Case 6.-Patient a doctor; uvulotomy performed December 17th, 1930, on account of cough. Reported June 11th, 1931, "No irritating cough; your partial removal of uvula has been distinctly successful.

Case 7.-Tickling cough of two months' duration. Tip of uvula removed January 6th, 1931. Reported next day, cough much better.

Case 8. - Tip of uvula removed June 17th, 1931, on account of "choking" feeling. A month later reported quite relieved. Case 9.-Tip of uvula removed July 1st, 1931, on account of cough on lying down. Reported November 25th, 1931, cough much better for a month and still much less than before operation; can now lie down without coughing. Patient has bronchitis.

Case 10.-Tickling cough at night or in a warm room. Elongated uvula shortened October 19th, 1931; reported October 27th, 1931, cough gone.

Case 11.-Elderly man, bleeder, tickling cough at night four months. Trailing uvula shortened with scissors, galvanocautery to raw surface, December 8th, 1931 ; no haemorrhage. Reported a week later, and recently, cough quite gone, " a great success."

My colleague Dr. P. H. Abercrombie, writing on September 26th, 1931, reminded me that many years ago I shortened his uvula with the "very greatest relief" to him. "My trouble was an irritating spasmodic cough on lying down in bed on my back; more than once it resembled a mild attack of whooping-cough. After thoroughly cocainizing, you removed fully half an inch of my uvula, and the cough has never bothered me since! You may add my name to your list, if you like, and I can honestly say that I have quite a number of times relieved and removed an irritating cough by shortening an elongated uvula."

Most of us have seen cases of inordinately long uvulas which appeared to nestle down quietly without causing any symptoms whatever; to shorten these would serve no good purpose. As a rule Dr. Feldman's proviso is appropriate-that the operation is called for only when there is no other apparent cause for the cough, such as tuberculosis. There are exceptions, however, as, for example, in a few cases of tuberculosis of the larynx and lungs, in which the exciting cough is increased in intensity and in frequency by the presence of a trailing uvula, with its characteristic symptum of tickling cough on lying down. In such cases I have diminished the suffering very greatly by discreet and almost bloodless shortening of the uvula. Another exceptional case was one of ictus laryngis, McBride's laryngeal syncope, commonly misnamed "laryngeal vertigo." The patient was a middleaged man suffering from cancer of the inlet of the larynx, with narrowing of the air-way. On several occasions he had dropped down at the commencement of a cough. I found an elongated uvula, the presence of which excited the cough. I therefore shortened it, and during the brief remainder of his life the patient was free from cough and attacks of syncope.

A word or two on the little operation may not be out of place. After anaesthetization of the uvula a pair of scissors are held in the right hand and a forceps in the left. The scissors are used to depress the tongue while the tip of the uvula is gripped with the forceps and pulled forwards. The scissors are then used to cut through the uvula upwards and backwards so as to leave the raw surface on the posterior aspect of the organ. The portion left should be of the size of a small normal uvula. The ring end of the scissors may with advantage be curved slightly downwards so that the knuckles of the right hand may not obstruct the view, and the distal end may be very slightly curved upwards. It is very helpful, however, to have sharp points on the grasping parts of the forceps, as the uvula is most elusive, especially when the tongue wobbles actively.

The ideal result is obtained if the removal of a small semi-oedematous elongation beyond the muscle is sufficient to produce the desired effect, but this is only occasionally possible. As a counsel of perfection it has been proposed to cut a wedge out of the tip and bring the little flaps of mucous membrane together with a stitch. Under ordinary circumstances we have to be content with a less elaborate technique. I find it very desirable to minimize the chance of haemorrhage by applying the galvano-cautery to the raw surface, and I have amputated by means of the galvano-caustic snare, though without any very special advantage. In respectful dissent from the opinion of those who hold that calcium is effective only if administered by the veins, I have found it advisable to prescribe calcium bromide before meals and at bedtime for the two or three days preceding the operation, to quiet the nerves and increase the coagulability of the blood.

If these remarks encourage the practitioner to shorten uvulas in appropriate cases, as of old, I am sure much relief will be afforded which is otherwise withheld.

\section{DIVERTICULUM OF THE MALE URETHRA}

With a Suggestion as to its Origin

BY

C. HOPE CARLTON, M.CH.Oxon, F.R.C.S.ENG.

SURGEON, ROYAL ALBERT DOCK HOSPITAL ; SURGICAL REGISTRAR, LONDON TEMPERANCE HOSPITAL

This condition is rare, there being about one hundred cases described in the literature. Many hospitals see, perhaps, one case in five or ten years.

It has been the custom to distinguish between cases supposed to have a congenital origin (although the diverticulum does not show itself until quite late in life) and cases which are acquired as the result of urethral obstruction or trauma. The explanation offered of the congenital diverticulum is that at birth there is a flaplike obstruction where that part of the urethra which arises in the cloaca joins with the part which was formed in the external genitalia. It is supposed that in a few hours increasing urinary pressure overcomes this obstruction, but there is left a tiny potential diverticulum. Later, in middle life, for some obscure reason, this sac suddenly enlarges and becomes a clinical entity. Now this theory puts an undue strain on the imagination. It resembles other embryological hypotheses made in the early days before research had placed this science on its modern foundation. Such a pioneer hypothesis was that which accounted for the downgrowth of Meckel's cartilage by manual traction exerted by the foetus.

The second class of urethral diverticulum is easier to understand. The acquired obstruction of a stricture or stone causes the proximal urethra to dilate, and later to form a lateral expansion; or a break in the wall of the urethra may result in extravasation of urine into a space which becomes lined with epithelium; or a peri-urethral abscess or extraneous cyst may burst into the urethra ; or there may be a pressure dilatation of Cowper's gland or of the uterus masculinus. Finally, after perineal prostatectomy, the cavity from which the prostate was removed may dilate and present in the perineum. Its passage forward is prevented by the triangular ligament.

\section{Diagnosis of Urethral Diverticulum}

This is often made during the investigation of other conditions, which themselves gave rise to the diverticulum. Should the diverticulum dominate the clinical picture, the landmarks are a fluid swelling, associated with frequent micturition, poor stream, and perineal discomfort. The swelling is perineal, scrotal, or penile. The frequency is of the type of a dribble when seated or lying down, or on standing or walking: 


\section{Discussion of a Recent Case}

The above summary of etiology and diagnosis is given, since there will now be described a patient to whom none of the mentioned causes of origin appear to apply, and in whom diagnosis was made only during operation.

A man, aged 66 , a road sweeper by occupation, presented himself in January, 1929, with the symptoms and signs of benign enlargement of the prostate. This was enucleated in the usual manner by the suprapubic route in a one-stage operation, use being made of a Pilcher bag, which was removed forty-eight hours later. This bag was maintained in position by a cord passing over a puliey to a weight of $1 \mathrm{lb}$. Five days later severe bleeding occurred, which was controlled by reintroducing the bag for several hours. The patient made a good recovery, and returned to his work as a roial sweeper.

During the next two years he had slight dribbling when sitting down, and during this period developed a right inguinal hernia, which filled half the scrotum when standing.

In May, 1931, there suddenly appeared a swelling the size of a lemon, in the left side of the scrotum. It was painless, but three days after it appeared he presented himself for examination. This showed an ordinary right-sided inguinal hernia, and the left-sided fluid swelling, which was apparently attached to the cord and taken to be a hydrocele. His complaint of dribbling was noted, but assumed to be an unfortunate complication of the prostatectomy of two years before. Urethroscopy and cystoscopy were not done, but he was admitted for scrotal exploration and repair of the hernia and hydrocele.

At the operation the hernia was dealt with in the orthodox manner and the left-sided swelling approached by an inguinal incision. The swelling was seen to be no part of the cord but was a fluid swelling arising by a pedicle one and a half inches wide from the corpus spongiosum, just in front of the bulb. A diagnosis of urethral diverticulum was made, and it was supposed that some distal obstruction accounted for it. A flexible bougie was therefore passed into the external meatus: It passed along the urethra with ease, and coiled itself up in the diverticulum. There was no urethral obstruction. The bladder was now opened suprapubically. Its neck presented the usual appearance at this time after prostatectomy. A sound entered at the internal meatus passed easily through, and could be manœuvred either into the diverticulum or onward to the external meatus. A soft rubber catheter was now introduced along the length of the urethra and the diverticulum opened. The neck seen from inside looked just like the neck of a diverticulum of the bladder. It was lined by smooth epithelium, and the orifice was one inch long and one-third of an inch wide, being aligned with the urethra, in which could be seen lying the catheter. The diverticulum was cut away, enough being left to close the pedicle over the catheter by double breasting. The catheter was tied in situ. Into the bladder was placed a Marion's tube. The left inguinal incision, through which the above urethral operation was performed, was closed. Apart from a scrotal haematocele the convalescence was uninterrupted, and the patient resumed his work as a road sweeper, on which he has since been engaged. When seen recently his only complaint was slight dribbling of urine, of which he loses several drops an hour.

\section{Observations on the Above Case}

Attention is drawn to the facility with which such a condition may be dealt with through a low inguinal incision. The lax scrotum resulting from the existing right-sided hernia made the exposure much easier. Further, this, like most other urethral operations, is best undertaken in association with suprapubic cystostomy, both for the additional angle of approach thus afforded to the lesion and for the suprapubic drainage during convalescence, which allows the urethra to remain dry throughout healing.

It appears advisable that disorders of micturition after prostatectomy should be investigated by urethroscopy and a urethrogram, as well as by cystoscopy.

When we come to consider the etiology of urethral diverticula in the light of this case it is by no means easy to arrive at any conclusion. But it may be said outright that the case affords no support for the theory of congenital origin. Why should this man, aged 66 , suddenly develop a urethral bulge because on the first day of his life there was a temporary rise of pressure in the bulbar urethra? Next, there was no doubt that the urethra distal to the diverticulum was completely patent at the time of the operation, and the obstruction of stricture or stone, which comes first to the mind, may be entirely dismissed.

It is a natural thing to try to associate this diverticulum with the prostatectomy he had undergone two years before. But it is very hard to do so. The prostatic cavity appeared normal, and was in no way connected with the diverticulum. Had it been, then the diverticulum would have presented in the perineum and not in the scrotum. There was no residual post-operative urethritis. Now it might have been that the passage of sounds after the prostatectomy had injured the urethra. But none of these had been passed for over two years.

I tentatively put forward the suggestion that there was urethral obstruction due to the right-sided inguinal bernia. The hernia pressed on the urethra just distal to the bulb. What would be the effect of this on a man with post-prostatectomy dribbling due to a damaged sphincter? It would help him to control the dribbling. But if he lay down or sat down, and the hernia went back up the inguinal canal, this additional control of micturition would cease. This is precisely what this man complained of. He could control his water when at work as a street sweeper, but not when at meals or in bed. Later, the pressure of the hernia on the urethra just in front of the bulb would cause obstruction, and behind this point the diverticulum appeared.

If this be the case, then we must regard urethral diverticulum as another of the possible complications of an inguinal hernia, as well as one of the differential diagnoses of hydrocele.

Finally, I would express my indebtedness to $\mathrm{Mr}$. James McClure, F.R.C.S., of the London Temperance Hospital, by whose kindness I had the opportunity of taking care of, and operating upon, this patient, and with whose permission these notes are published.

\section{HUMAN AND BOVINE TUBERCULOSIS} THE QUESTION OF MUTATION OF THE TUBERCLE BACILLUS

BY

NATHAN RAW, C.M.G., M.D., M.R.C.P. LORD CHANCELLOR'S VISITOR; LATE BRITISH MEMBER OF THE INTERNATIONAL COMMIITTEE ON TUBERCULOSIS

In my original paper in the British Medical Journal of 1903 I ventured on the following conclusions:

1. That there are two distinct varieties of tuberculosis affecting the human body, one produced by human tubercle, the other by bovine tubercle.

2. That human and bovine tubercle are separate and distinct.

3. That phthisis is caused by the human bacillus.

4. That gland, bone, and joint tuberculosis and lupus are of bovine origin and conveyed by milk.

The general opinion of bacteriologists with regard to specific micro-organisms is that they retain their characteristics under ordinary conditions, and are not subject to mutation. If, however, these micro-organisms are subjected to a new environment, in some instances a change takes place, which is usually called transmutation. I have for many years been of the opinion that bovine tubercle bacilli introduced into the human body in infancy or early childhood may, after many years' growth in new. 